\title{
Efficacy of percutaneous endoscopic gastrostomy on unplanned treatment interruption and nutritional status in patients undergoing chemoradiotherapy for advanced head and neck cancer
}

\author{
Masakazu Goda', Osamu Jinnouchi', Tsukasa Takaoka', Koji Abe ${ }^{1}$, Koich Tamura', Yutaka Nakaya², \\ Yoshihito Furukita ${ }^{3}$, Hirokazu Takechi ${ }^{3}$, Akira Tangoku ${ }^{3}$, and Noriaki Takeda ${ }^{1}$ \\ ${ }^{1}$ Department of Otolaryngology, ${ }^{2}$ Nutrition and Metabolism and ${ }^{3}$ Surgery, University of Tokushima School of Medicine, Tokushima, Japan
}

\begin{abstract}
Objective : Efficacy of percutaneous endoscopic gastrostomy (PEG) on unplanned treatment interruption and nutritional status was examined in patients undergoing chemoradiotherapy (CRT) for advanced head and neck cancer. Methods : We retrospectively reviewed hospital charts of 44 patients with advanced head and neck cancer who were treated with CRT. Results : CRT-induced mucositis of grade 3 or worse and inadequate oral intake of less than one third of their usual intake developed in 33 patients who were recommended PEG placement, but not in 11 patients. Thirteen patients accepted PEG placement and then completed CRT (compliant group). However, among 20 patients who refused both PEG and nasogastoric tube (NGT) placements (non-compliant group), 10 required unplanned interruptions of CRT at a radiation dose around 30-40 Gy (UICRT group) while 10 others could complete CRT without interruption (C-CRT group) CRT. Total serum protein levels were significantly decreased after CRT in all patients. Discussion : It is suggested that therapeutic PEG placement is useful for preventing unplanned interruption of CRT in patients with advanced head and neck cancer. After severe mucositis and inadequate oral intake have developed during CRT, PEG placement should be considered before the radiation therapy dose of 30 Gy. J. Med. Invest. 62 : 173-176, August, 2015
\end{abstract}

Keywords : percutaneous endoscopic gastrostomy ; PEG ; nutritional support; chemoradiotherapy ; head and neck cancer

\section{INTRODUCTION}

For patients with advanced stage squamous cell carcinoma of the head and neck, chemoradiotherapy (CRT) is frequently used as the primary treatment in order to achieve organ preservation and to improve survival. However, most patients who are treated with CRT for advanced head and neck cancer suffer from its side effects such as dysphagia, mucositis, xerostomia and nausea. Severe oropharyngeal mucositis leads to decreased oral food intake, resulting in interruption of CRT that may reduce its efficacy $(1,2)$.

Nasogastoric tube (NGT) is often placed as enteral nutritional support for head and neck cancer patients with CRT-induced mucositis. However, NGT is frequently associated with local irritation, which might worsen mucositis, risk of aspiration pneumonia and high frequency of occlusion. Recently, percutaneous endoscopic gastrostomy (PEG) has been introduced for nutritional support for patients with advanced head and neck cancer undergoing CRT $(3,4)$. It was reported that in comparison with NGT, PEG was equally effective in maintaining body weight with less rates of mechanical failure and aspiration pneumonia (5). Moreover, PEG has additional advantages in term of mobility, cosmetics and quality of life. Thus, PEG is now a safe and effective method of providing enteral nutrition during CRT in patients with advanced head and neck cancer.

In order to clarify if PEG placement improves the completion of CRT and nutrition status in head and neck cancer patients, the

Received for publication December 4, 2014 ; accepted February 20, 2015.

Address correspondence and reprint requests to Masakazu Goda, M.D. Department of Otolaryngology, University of Tokushima School of Medicine 3-18-15 Kuramoto, Tokushima 770-8503, Japan and Fax : +81-88633-7170. present study examined the efficacy of PEG on unplanned treatment interruption and total serum protein levels in patients treated with CRT for advanced head and neck cancer. For that purpose, we recommended the placement of PEG in patients with inadequate oral food intake of less than one third of their usual intake due to severe CRT-induced mucositis. Then, we compared the rate of treatment interruption and total serum protein concentration in patients who accepted PEG placement with those who refused it.

\section{PATIENTS AND METHODS}

Patients and study design

We retrospectively reviewed hospital charts of 44 patients with advanced head and neck cancer (38 males, 6 females, the average age was $68.4 \pm 10.3$ years old, 53 -85 years old) who were treated with CRT at the Department of Otolaryngology of Tokushima University Hospital. Tumor sites included : nasopharynx $(n=7)$, oropharynx $(n=8)$, hypopharynx $(n=13)$, larynx $(n=10)$, oral cavity $(n=4)$, and metastatic cervical lymph nodes of unknown origin $(n=2)$. All patients were previously untreated and had locally advanced stage III or stage IV disease (Table 1). The radiation dose was approximately $70 \mathrm{~Gy}$, and chemotherapy usually consisted of cisplatin and 5-fluorouracil. We performed concurrent CRT, except alternative CRT for nasopharyngeal cancer. This study was approved by the Committee for Medical Ethics of Tokushima University Hospital.

\section{Treatment and placement of PEG}

Indications criteria for PEG placement were as follows : CRTinduced mucositis of grade 3 or worse on common terminology criteria for adverse events (CTCAE Version 4.0) and inadequate 
Table 1 Patient characteristics

\begin{tabular}{lrrcccc}
\hline Tumor site & $\mathrm{n}$ & $\begin{array}{c}\text { Mean age } \\
\text { (years) }\end{array}$ & Male & Female & Stage III & Stage IV \\
\hline Nasopharynx & 7 & $68.9 \pm 14.0$ & 6 & 1 & 4 & 3 \\
Oropharynx & 8 & $63.1 \pm 9.9$ & 6 & 2 & 2 & 6 \\
Hypopharynx & 13 & $69.3 \pm 11.9$ & 12 & 1 & 2 & 11 \\
Larynx & 10 & $67.6 \pm 7.6$ & 10 & 0 & 5 & 5 \\
Oral cavity & 4 & $74.8 \pm 6.2$ & 4 & 0 & 1 & 3 \\
unknown origin & 2 & $77.5 \pm 9.2$ & 0 & 2 & & \\
\hline Total & 44 & & 38 & 6 & &
\end{tabular}

Data show as either $n$, or mean \pm standard deviation.

oral intake of less than one third of usual intake during CRT. This is because in patients with CRT-induced mucositis of grade 3 , after their nutritional intake becomes less than one third of usual intake, protein catabolism is enhanced resulting in delayed healing of mucositis and exacerbation of patient's malnutrition (6-9). Thirty three patients who met the criteria for PEG placement during CRT were recommended to be managed by this method for nutritional support. Among them, 13 patients accepted PEG placement (the compliant group), which was performed by surgeons at the Department of Surgery of Tokushima University Hospital. On the other hand, 20 patients refused both PEG and NGT placements (the non-compliant group), while the remaining 11 patients did not meet the criteria for PEG placement (non-indication group).

PEG was placed using the Pull method, as described by Ponsky et al. (10). No complications were associated with PEG tube placement such as local infections, tube blockage, migration or dislodgement.

CRT-induced non-hematologic toxicity of grade 3 or worse (other than mucositis) and CRT-induced hematologic toxicity of grade 4 led to the interruption of CRT. But, in patients with only CRT-induced mucositis of grade 3 or worse without hematologic toxicity of grade 4, CRT was interrupted when patient had difficulty eating.

\section{Assessment of total serum protein levels}

Total serum protein (TP) levels were measured before and after CRT in all patients and taken as an index of nutritional status.

\section{Statistical analysis}

Fisher's exact probability test, Student's $t$-test and ANOVA with Kruskal-Wallis post hoc test were used for statistical analysis, and $P<0.05$ was considered significant.

\section{RESULTS}

Efficacy of PEG on treatment interruption of chemoradiotherapy

All 44 patients who were treated with CRT for advanced head and neck cancer suffered from CRT-induced mucositis of grade 3 or worse and 33 of them developed inadequate oral intake of less than one third of their usual intake. Because they met our criteria for PEG placement during CRT, we recommended them for this nutritional support method. Among them, 13 patients accepted PEG placement (the compliant group) and then completed CRT. However, 20 patients refused both PEG and NGT placements (the non-compliant group). Thereafter, unplanned interruptions of CRT were required in 10 patients who had difficulty eating (Fig. 1). The interruption rate was $50 \%$ in non-compliant group, a significant increase compared with that of the compliant group (Table 2). In the non-compliant group, CRT interruption periods were 6 to 35 days (10.5 \pm 8.8 days) and reached $80 \%$ in most of them, while CRT

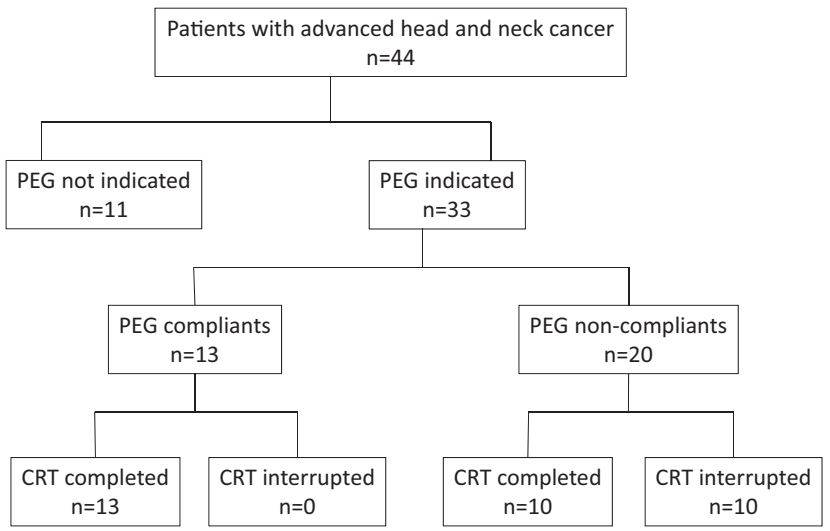

Fig. 1 : Patients flowchart. PEG : percutaneous endoscopic gastrostomy, CRT : chemoradiotherapy.

Table 2 Interruption rate of chemoradiotherapy in patients with advanced head and neck cancer

\begin{tabular}{lc}
\hline PEG placement & Interruption rate of CRT \\
\hline Compliant group & $0 \%(0 / 13)$ \\
Non-compliant group & $50 \% *(10 / 20)$ \\
Non-indication group & $0 \%(0 / 11)$ \\
\hline
\end{tabular}

$* \mathrm{p}<0.01$ vs. compliant and non-compliant patients group.

PEG : percutaneous endoscopic gastrostomy, CRT : chemoradiotherapy.

was interrupted at a radiation dose around 30-40 Gy (Fig. 2). The other 10 non-compliant patients could complete CRT without interruption. The remaining 11 patients of the non-indication group also completed CRT without interruption.

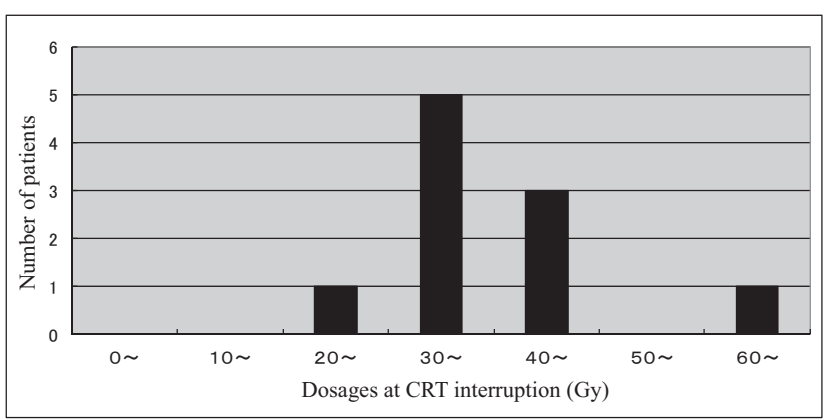

Fig. 2 : Doses of radiation at interruption of CRT in non-compliant patients with advanced head and neck cancer. PEG : percutaneous endoscopic gastrostomy, CRT : chemoradiotherapy.

\section{Efficacy of PEG on nutritional status}

Serum TP levels were significantly decreased after CRT in each group (Fig. 3). The decrease rate of serum TP in the compliant group was $6.4 \pm 9.0 \%$ (mean $\pm \mathrm{SD}) ; 6.4 \% \pm 7.6$ in the non-compliant group ; $13.1 \pm 8.7 \%$ in patients who completed CRT without interruption, and $8.3 \pm 7.4 \%$ in those of the non-indication group. There were not significant differences among groups.

\section{Adverse events except mucositis during chemoradiotherapy}

In the compliant and non-compliant groups, 4 patients suffered 


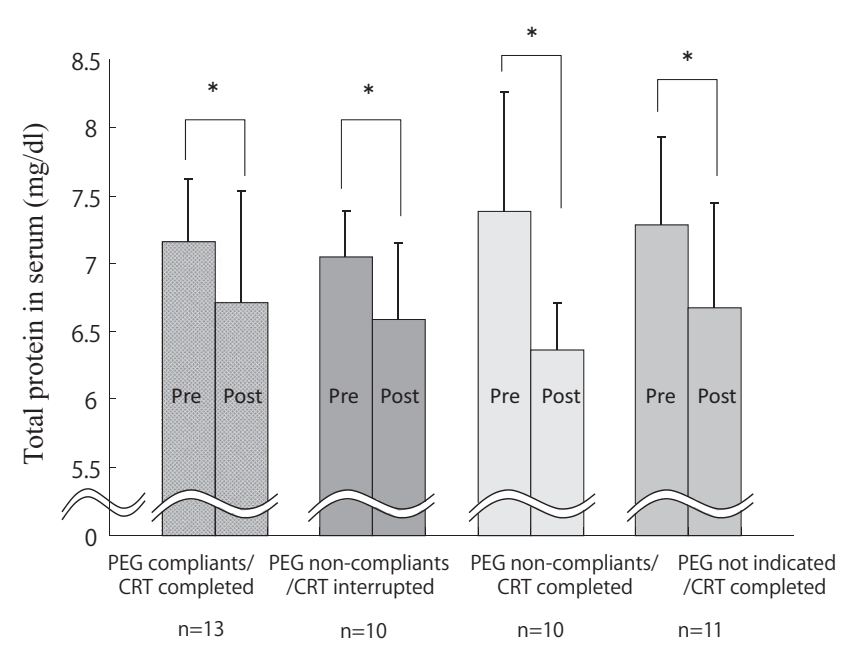

Fig. 3 : Changes in total serum protein levels in patients with advanced head and neck cancer after CRT. PEG : percutaneous endoscopic gastrostomy, CRT : chemoradiotherapy.

from grade 3 leukopenia in each group, while 1 patient suffered from both grade 4 leukopenia of and grade 3 dermatitis at the interruption of CRT. There was no significant difference in the frequency of grade 3 leukopenia between the groups.

\section{DISCUSSION}

Most patients with advanced head and neck cancer have some degree of malnutrition, which is deteriorated by toxic side-effects during CRT. Although NGT is useful for short-term nutritional support, PEG is indicated for long-term nutritional support when oral intake is inadequate. In the present study, of 44 patients who were treated with CRT for advanced head and neck cancer, 33 met the criteria for PEG placement. Among them, 13 patients accepted the placement and subsequently completed their CRT. However, among the 20 patients who refused both PEG and NGT placements, unplanned interruptions of CRT were required in 10 of them. Their interruption period ranged from 1 week to 4 weeks. Patel, et al. reported that advanced head and neck cancer patients who required break of radiation for more than 2 weeks were significantly more likely to have residual tumor in the neck after CRT (2). McCloskey, et al. also reported that CRT interruption of more than a week constitutes a significant higher risk of loco-regional recurrence of tumor (1). Therefore, it is suggested that PEG placement is useful for preventing unplanned interruption of CRT in patients with advanced head and neck cancer, resulting in improvement of their residual and recurrent rates of tumor. Prospective controlled studies are necessary to clarify whether PEG placement improves the prognosis of advanced head and neck cancer.

In the present study, among 10 patients who refused both PEG and NGT placements, unplanned interruptions of CRT were required at a radiation dose around $30-40 \mathrm{~Gy}$ in 8 patients. The indication of therapeutic PEG placement is still controversial, because no single marker can be used to identify malnutrition $(3,4)$. Therefore, after severe mucositis and inadequate oral intake have developed, therapeutic PEG placement should be considered before the dose of $30 \mathrm{~Gy}$ in patients with advanced head and neck cancer undergoing CRT.

The same as for patients in whom PEG placement was indicated, serum TP levels were significantly decreased after CRT in those who did not undergo the procedure. This finding suggests that malnutrition further developed even in patients with mild/moderate
CRT-induced mucositis during CRT. Moreover, it is suggested that therapeutic PEG placement did not prevent malnutrition, because TP levels were decreased even in patients who accepted it. All patients who accepted PEG placement completed CRT without interruption regardless of their significant decrease of serum TP levels after CRT. It may be suggested that enteral feeding by PEG placement improve nutritional status that can't be estimated by only serum TP levels. Since malnutrition is a strong independent predictor of survival in patients with advanced head and neck cancer (11), prophylactic PEG is recommended in all patients with advanced head and neck cancer before CRT.

Recently, a randomized controlled trial showed evidence for prophylactic PEG placement in the prevention of malnutrition and improved quality of life in patients with advanced head and neck cancer (12). Moreover, Raykher reported that PEG feeding allowed CRT to continue without interruption in $93 \%$ of patients with head and neck cancer (13). However, Lee et al. reported that prophylactic PEG had no effect in the rate of treatment interruption in patients undergoing intensive radiation therapy for head and neck cancer (14). In the present study, based on our indication criteria for of PEG placement including CRT-induced mucositis of grade 3 or worse and inadequate oral intake of less than one third of the usual intake, 11 patients $(25 \%)$ did not meet our indication criteria, but completed CRT uninterrupted without PEG. Therefore, it is suggested that prophylactic PEG placement is not needed before CRT in all head and neck cancer patients and therapeutic PEG placement is preferred in response to inadequate oral intake during CRT. Further study is necessary to examine whether prophylactic or therapeutic PEG placement is warranted in the treatment of advanced head and neck cancer by CRT (15).

The pull method of PEG placement in patients with head and neck cancer is widely applied, because it is easy to perform and can be accomplished without general anesthesia, though there is a possibility of exit site metastasis due to the pull method. However, the risk is accepted, because it is very low $(<1 \%)(16)$.

There is also the possibility that PEG placement during CRT increases the risk of surgical site infection and bleeding of CRTinduced mucositis. To reduce the risk, PEG was placed more than a week before or after chemotherapy in the study. Accordingly, no surgical complications developed.

In conclusion, in patients undergoing CRT for advanced head and neck cancer, the efficacy of PEG on its unplanned interruption was examined. Therapeutic PEG placement was useful in preventing unplanned interruption of CRT. After severe mucositis and inadequate oral intake have developed during CRT, PEG placement should be considered before the dose of $30 \mathrm{~Gy}$.

\section{ACKNOWLEDGEMENT}

We thanks all members of nutrition supporting team of Tokushima University Hospital for their cooperation.

\section{CONFLICT OF INTEREST}

The authors declare no conflict of interest.

\section{REFERENCES}

1. McCloskey SA, Jaggernauth W, Rigual NR, Hicks WL, Popat SR, Sullivan M, Mashtare TL, Khan MK, Loree TR, Singh $\mathrm{AK}$ : Radiation treatment interruptions greater than one week and low hemoglobin level $(12 \mathrm{~g} / \mathrm{dL})$ are predictors of local regional failure after definitive concuurent chemotherapy and 
untesity-modulated radiation therapy for squamous cell carcinoma of the head and neck. Am J Clin Oncol 32 : 587-591, 2009

2. Patel UA, Patadia MO, Holloway N, Rosen F : Poor radiotherapy compliance predicts persisitent regional disease in advanced head/neck cancer. Laryngoscope 119 : 528-533, 2009

3. Colasanto JM, Prasad P, Nash MA, Decker RH, Wilson LD : Nutritional support of patients undergoing radiation therapy for head neck cancer. Oncol 19 : 371-379, 2005

4. Raykher A, Russo L, Schattner M, Schwartz L, Scott B, Shike $\mathrm{M}$ : Enteral nutrition support of head and neck cancer patients. Nutr Clin Pract 22 : 68-73, 2007

5. Magne N, Marcy PY, Falewee MN, Schneider M, Demard F, Bensadoun RJ : Comparison between nasogastric tube feeding and percutaneous fluoroscopic gastrostomy in advanced head and neck cancer. Eur Arch Otolaryngol 258 : 89-92, 2001

6. Nguyen NP, North D, Smith HJ, Dutta S, Alfieri A, Karlsson U, Lee H, Martinez T, Lemanski C, Nguyen LM, Ludin A, Sallah S : Safety and effectiveness of prophylactic gastrostomy tubes for head and neck cancer patients undergoing chemoradiation. Surgical Oncology 15(4) : 199-203, 2006

7. Singer P, Berger MM, Van den Berghe G, Biolo G, Calder P, Forbes A, Griffiths R, Kreyman G, Leverve X, Pichard C : ESPEN Guidelines on Parenteral Nutrition : intensive care. Clinical Nutrition 28(4) : 387-400, 2009

8. Arends J, Bodoky G, Bozzetti F, Fearon K, Muscaritoli M, Selga G, van Bokhorst-de van der Schueren MA, von Meyenfeldt M ; DGEM (German Society for Nutritional Medicine), Zürcher G, Fietkau R, Aulbert E, Frick B, Holm M, Kneba M, Mestrom HJ, Zander A ; ESPEN (European Society for Parenteral and Enteral Nutrition) : ESPEN Guidelines on Enteral Nutrition : Non-surgical oncology. Clinical Nutrition 25(2) : 245-259, 2006
9. Arnold M, Barbul A : Nutrition and wound healing. Plastic and Reconstructive Surgery 117 : 42S-58S, 2006

10. Ponsky JL, Gauderer MW, Stellato TA : Percutaneous endoscopic gastrostomy. Review of 150 cases. Arch Surg 118 : 913914, 1983

11. Mick R, Vokes EE, Weichselbaum RR, Panje WR: Prognostic factors in advanced head and neck cancer patients indergoing muitimodality therapy. Otolaryngol Head Neck Surg 105 : 62-73, 1991

12. Silander E1, Nyman J, Bove M, Johansson L, Larsson S, Hammerlid E : Impact of prophylactic percutaneous endoscopic gastrostomy on malnutrition and quality of life in patients with head and neck cancer : a randomized study. Head Neck 34 : 1-9, 2012

13. Raykher A, Correa L, Russo L, Brown P, Lee N, Pfister D, Gerdes H, Shah J, Kraus D, Schattner M, Shike M : The role of pretreatment percutaneous endoscopic gastrostomy in facilitating therapy of head and neck cancer and optimizing the body mass index of obase patient. JPEN J Parenter Enteral Nutr 33 : 404-410, 2009

14. Lee JH1, Machtay M, Unger LD, Weinstein GS, Weber RS, Chalian AA, Rosenthal DI : Prophylactic gastrostomy tubes in patients undergoing intensive irradiation for cancer of the head and neck. Arch Otolaryngol Head Neck Surg 124 : 871-875, 1998

15. Locher JL1, Bonner JA, Carroll WR, Caudell JJ, Keith JN, Kilgore ML, Ritchie CS, Roth DL, Tajeu GS, Allison JJ : Prophylactic percutaneous endoscopic gastrostomy tube placement in treatment of head and neck cancer : a comprehensive review and call for evidence-based medicine. JPEN J Parenter Enteral Nutr 35 : 365-374, 2011

16. Hunter JG. Tumor implantation at PEG exit sites in head and neck cancer patients : how much evidence is enough? J Clin Gatroenterol 37 : 280, 2003 